\title{
Container/Closure Labeling
}

National Cancer Institute

\section{Source}

National Cancer Institute. Container/Closure Labeling. NCI Thesaurus. Code C113016.

The action of affixing an identifier to a container or closure of an item. 